\title{
Procesamiento de Arvejas (Pisum sativum L.). Parte 3: Cinética de Pérdida de Agua en Chips de Arveja en Condiciones de Fritura Convencional y a Vacío
}

\author{
Oswaldo Osorio(1), Gloria Rodríguez ${ }^{(2)}$, Francisco Castellanos ${ }^{(3)}$, Andrés Chávez $^{(3)}$ \\ (1) Universidad de Nariño, Facultad Ingeniería Agroindustrial, Grupo Investigación GAIDA, Calle $18 \mathrm{Cr}$ \\ 50, sede universitaria Torobajo, San Juan de Pasto - Colombia, (e-mail: osorio_oswaldo@hotmail.com). \\ (2) Universidad de San Buenaventura- Av. 10 de Mayo, Cali-Colombia (e-mail: gcrodrig@usbcali.edu.co). \\ (3) Universidad de Caldas, Calle 65 No 26 - 10, Caldas- Colombia. \\ (e-mail: francisco.castellanos@ucaldas.edu.co; andres.chavez@ucaldas.edu.co).
}

Recibido Ago. 25, 2015; Aceptado Oct. 10, 2016; Versión final Mar. 10, 2016, Publicado Ago. 2016

\section{Resumen}

Se determinó la cinética de pérdida de agua en chips de arveja (Pisum sativum L.) de la variedad Obonuco Andina en diferentes condiciones de fritura a presión atmosférica y de vacío. Se consideraron tres presiones $\left(78,43\right.$ y $9 \mathrm{kPa}$ ) asociadas a tres temperaturas $\left(140,160\right.$ y $\left.180{ }^{\circ} \mathrm{C}\right)$, y se utilizó una relación semilla/aceite $1: 8(\mathrm{p} / \mathrm{v})$, tomando mediciones entre 0 y 240 segundos. El efecto de la presión y la temperatura fue evaluado mediante el coeficiente de difusión (Deff) y la energía de activación (Ea), mientras que las cinéticas de pérdida de agua fueron estudiadas con tres modelos (Newton, HendersonPabis y Page). Se determinó dependencia de la difusividad con la temperatura y la presión. La ecuación de Arrhenius dio valores de la energía de activación Ea de 18.67, 13.82 y $13.75 \mathrm{~kJ} / \mathrm{mol}$, para 78, 43 y 9 $\mathrm{kPa}$ respectivamente. Los modelos expuestos describen adecuadamente la cinética de pérdida de agua.

Palabras clave: pérdida de agua; difusividad; energía de activación; constante cinética; Pisum sativum L.

\section{Kinetics of Water Loss in Chips of Peas (Pisum Sativum L.) by Conventional and Vacuum Frying Conditions}

\begin{abstract}
The kinetics of water loss from chips peas (Pisum sativum L.) of Andina Obonuco variety in different frying conditions at atmospheric pressure and vacuum was determined. Three pressures were considered (78, 43 and $9 \mathrm{kPa})$ associated at three temperatures $\left(140,160\right.$ and $\left.180^{\circ} \mathrm{C}\right)$, and a seed/oil ratio $1: 8(\mathrm{w} / \mathrm{v})$ was used taking measurements between 0 and 240 seconds. The effects of pressure and temperature were evaluated by the diffusion coefficient (Deff) and the activation energy (Ea), while the kinetics of water loss was studied with three models (Newton, Henderson-Pabis and Page). The dependence of diffusivity on temperature and pressure was determined. The Arrhenius equation gave values for the activation energy Ea of $18.67,13.82$ and $13.75 \mathrm{~kJ} / \mathrm{mol}$ at 78,43 and $9 \mathrm{kPa}$ respectively. The models analyzed in this work adequately describe the kinetics of water loss.
\end{abstract}

Keywords: water loss; diffusivity; activation energy; kinetic constant; Pisum sativum L. 


\section{INTRODUCCIÓN}

La arveja (Pisum sativum L.) es uno de los principales cultivos comestibles en el mundo (Amarakoon et al., 2012). En Colombia es una de las leguminosas de mayor importancia en especial en el Sur del país, siendo el departamento de Nariño, uno de los mayores productores (Osorio y Castaño, 2011), allí se cultiva desde los 1700 a 3100 metros de altitud, reflejando una alta capacidad de adaptación, alto potencial de rendimiento y posibilidad de cosechar el grano en fresco o en seco (Fenalce, 2010). Según Pabón y Castaño (2012) lo representativo de este cultivo, es que se lleva a cabo por pequeños y medianos agricultores, generando un factor estabilizador en la economía del campesino y en la seguridad alimentaria. No obstante, a pesar de Nariño ser unos de los mayores productores de arveja, aun se evidencia un incipiente manejo post-cosecha, y un nulo nivel de transformación agroindustrial, lo cual ha ocasionado pérdidas de hasta un $42.1 \%$ del total de la producción, atribuidas al igual que en otros productos al uso de tecnologías inapropiadas, manipulación inadecuada, falta de conocimiento e inversión en postcosecha (Espinosa et al., 2010) factores que no garantizan periodos prolongados de vida útil ocasionando detrimento en las características fisicoquímicas, nutricionales y sensoriales, producto de la acción de distintos microorganismos, procesos fisiológicos propios de las semillas, elevada actividad de agua (Garg et al., 2014) entre otras.

Lo anterior condujo a que Osorio (2011) señalará la necesidad de alternativas agroindustriales para redirigir al material vegetal y así optar por minimizar las pérdidas durante la etapa post-cosecha. Dentro de las alternativas de conservación la deshidratación se destaca; la reducción de la humedad previene el crecimiento de microorganismos y minimiza las reacciones de deterioro (Téllez-Pérez et al., 2012), sin embargo, el producto en estas condiciones no es apto para consumo; conduciendo a la necesidad de plantear alternativas que reduzcan el contenido de agua de las semillas y al tiempo confiera características organolépticas deseables.

Por ello, la fritura es una de las opciones dentro de un proyecto regional, que busca estudiar el efecto de las condiciones de operación sobre diferentes fenómenos que se describen más adelante. Según Alvis, Villada y Villada (2008) este es uno de los procedimientos más populares para preparar alimentos; usando la cocción en aceite o grasa caliente a temperaturas elevadas, donde el aceite actúa como transmisor del calor produciendo un calentamiento rápido y uniforme en el alimento (Kochhar y Gertz, 2004); durante este proceso se presentan fenómenos como la pérdida de humedad, ganancia de aceite, desnaturalización de las proteínas, gelatinización de los almidones y cuantiosos cambios microestructurales en el interior del alimento (Alvis y Vélez, 2008; Vitrac, Trystram y Raoult, 2003). Cambios atribuidos a factores como: la humedad inicial del alimento, la calidad del aceite, la temperatura del proceso y el tiempo de residencia del producto en el aceite caliente (Dobarganes, Márquez y Velasco, 2000); que de no ser controlados influirán en la calidad del producto final.

En este sentido, dichos cambios, han conducido a utilizar alternativas como el uso de vacío, tratando de minimizar los efectos indeseados de la fritura convencional. Gómez (2012) menciona que el uso de vacío mejora la calidad de los productos fritos; productos como (chips de batata, chips de manzana, chips de papa azul) presentaron menor absorción de aceite (menos sabor a grasa) con una mayor retención del color y de sus sabores naturales (Da Silva y Moreira, 2008). Pese a lo anterior aún son escasos los estudios de fritura de arveja (Pisum sativum L.) más aun con el uso de vacío.

Costa y Olivera (1999) mencionan que para modelar este proceso, se deben tener en cuenta los dos principales fenómeno que en esta operación se producen, la remoción del agua y la absorción de aceite, ello permitirá modelar y controlar el proceso. En el estudio presentado por Brousse et al., (2012) se evidencia la existencia de modelos teóricos y empíricos para la predicción y la optimización de fenómenos de transferencia de masa como los observados en la fritura. Banga, Balsa, Moles y Alonso (2003) mencionan que el modelamiento matemático es una herramienta básica en los procesos industriales, puesto que permite realizar la estimación de su desarrollo con anticipación, lo que conllevará a controlar la calidad y mejorar los procesos. Dentro de este contexto, el objetivo general de este estudio fue modelar la pérdida de humedad en chips de arveja (Pisum sativum L.) de la variedad Obonuco Andina en condiciones de fritura a vacío.

\section{MATERIALES Y MÉTODOS}

\section{Material de estudio}

Se utilizaron semillas de arvejas (Pisum sativum L.) de la variedad Obonuco Andina, el material se recolectó al quinto mes de establecido el cultivo (exclusivo para ensayos de posibilidades industriales de arveja) en la granja del Centro Internacional de Producción Limpia LOPE, Sena Regional Nariño, ubicado a 2650 msnm, 
temperatura promedio $13 \stackrel{\circ}{\circ}$ y precipitación promedio anual de $700 \mathrm{~mm}$, condiciones favorables para el cultivo. El material se cosechó en un periodo no superior a 24 horas antes de realizar los ensayos, posteriormente la arveja se envió a la ciudad de Manizales a la Unidad de Tecnología de Alimentos de la Universidad de Caldas-Colombia, en donde se desgranó y se inspeccionó según parámetros establecidos en la Norma técnica Colombiana NTC 1250 (1979); la humedad promedio de las semillas se encontró entre 66 y 68 \% (base húmeda), el diámetro promedio de las semillas fue mayor a $9 \mathrm{~mm}$.

\section{Proceso de fritura}

Cada unidad experimental se constituyó por $100 \mathrm{~g}$ de arveja fresca, el proceso se llevó a cabo en batch, en un prototipo para fritura construido por la Unidad de Tecnología de Alimentos de la Universidad de CaldasColombia equipo con una capacidad de 7 litros que consta de tres termocuplas para registrar temperaturas internas en la matriz de estudio, un tablero de control automatizado, una bomba de vacío y software a la medida capaz de controlar y registrar las condiciones de trabajo necesarias; se empleó aceite $100 \%$ vegetal industrial, con punto de humo de $230{ }^{\circ} \mathrm{C}$ (Norma Técnica 3272, 1997), la relación producto/aceite se mantuvo constante $1: 8(\mathrm{p} / \mathrm{v})$.

Se evaluaron tres presiones asociadas a tres temperaturas: T1a $(78 \mathrm{kPa}$ presión atmosférica aproximada de Manizales $\left.\times 140{ }^{\circ} \mathrm{C}\right), \mathrm{T} 1 \mathrm{~b}\left(78 \mathrm{kPa} \times 160^{\circ} \mathrm{C}\right)$, $\mathrm{T} 1 \mathrm{c}\left(78 \mathrm{kPa} \times 180^{\circ} \mathrm{C}\right), \mathrm{T} 2 \mathrm{a}\left(43 \mathrm{KPa} \times 140{ }^{\circ} \mathrm{C}\right), \mathrm{T} 2 \mathrm{~b}(43 \mathrm{KPa} \times$ $\left.160^{\circ} \mathrm{C}\right)$, T2c $\left(43 \mathrm{kPa} \times 180^{\circ} \mathrm{C}\right)$, T3a $\left(9 \mathrm{kPa} \times 140^{\circ} \mathrm{C}\right)$, T3b $\left(9 \mathrm{kPa} \times 160^{\circ} \mathrm{C}\right)$, T3c $\left(9 \mathrm{kPa} \times 180^{\circ} \mathrm{C}\right)$ por tiempos de fritura de entre 0 y 240 segundos, para un total de nueve curvas de cinética de pérdida de humedad que se llevaron a cabo por triplicado. Una vez culminado cada ensayo de fritura en los ensayos por debajo de $78 \mathrm{Kpa}$, se realizó la restitución de la presión atmosférica, posteriormente se retiró la tapa hermética y se eliminó el exceso de aceite superficial con papel absorbente según la metodología descrita por Bravo (2008).

\section{Determinación humedad}

Se determinó según la metodología descrita por Bernal (1998). Se pesaron $10.00 \pm 0.10 \mathrm{~g}$ de cada tratamiento, aparte de ello se pesó una capsula de porcelana previamente seca al calor. Se calentó a 105.0 $\pm 0.10^{\circ} \mathrm{C}$ en el horno eléctrico, por un tiempo de 24 horas hasta lograr un peso constante. Se enfrió y se pesó; los resultados se expresaron en base húmeda.

\section{Estudio de la pérdida de agua como modelo difusivo}

Se utilizó la metodología descrita por Melquíades et al., (2009) a partir de la segunda Ley de Fick, ecuación de difusión, con geometría de un cilindro (ecuación 1).

$$
\frac{\mathrm{m}-\mathrm{ms}}{\mathrm{mo}-\mathrm{ms}}=\frac{4}{5.783} \exp \left(\frac{5.783 \text { Def } \mathrm{t}}{\mathrm{R}^{2}}\right)
$$

En esta ecuación, Def es el coeficiente de difusión de agua en el grano; t es el tiempo; mo es la humedad inicial; $\mathrm{ms}$ es la humedad en equilibrio y $\mathrm{R}$ es el radio de la semilla. En el mismo sentido, se determinó la energía de activación (Ea), de la operación de fritura, a partir del coeficiente de difusión que se ajusta a la ecuación de Arrhenius debido a su dependencia con la temperatura (Melquíades et al., 2009) ecuación 2.

$$
\operatorname{Def}=\text { Do } \exp ^{\left(-\frac{E a}{R T}\right)}
$$

En esta ecuación, Do es el factor pre-exponencial; Ea es la energía de activación ( $\mathrm{kJ} / \mathrm{mol})$ y $\mathrm{R}$ es la constante de los gases (8.314 J/g mol K).

Modelado matemático de las curvas de pérdida de agua

Se determinó mediante los modelos de (Newton, Henderson - Pabis y Page) expuesto por Vega, Tello y Lemus (2007), Martínez et al., (2010) y Da Rocha et al., (2012) ecuaciones 3, 4 y 5 respectivamente.

$$
\begin{aligned}
& M R=\exp ^{-K^{*} t} \\
& M R=a^{*} \exp ^{-K^{*} t} \\
& M R=\exp ^{-K^{*} t^{n}}
\end{aligned}
$$


Donde: MR: se describe en la (6); K: constante de velocidad de pérdida de agua $\left(\mathrm{s}^{-1}\right)$; $\mathrm{t}$ : tiempo de pérdida de agua en la fritura (s); a y $\mathrm{n}$ : constantes de los modelos. Los parámetros cinéticos $\mathrm{K}$ se obtuvieron a partir de los datos experimentales mediante regresión no lineal

$$
M R=\frac{X_{w t}-X_{w e}}{X_{w o}-X_{w e}}
$$

Donde: $X_{w t}$ : Contenido de agua al tiempo t (g de agua/g de sólido seco); $X_{w e}$ : contenido de agua en el equilibrio ( $\mathrm{g}$ de agua/g de solido seco) y $X_{w o}$ : Contenido de agua ( $\mathrm{g}$ de agua/g de sólido seco).

\section{Análisis de resultados}

Todos los resultados se expresaron como media más o menos la desviación estándar. Los gráficos fueron realizados con ayuda del programa Sigma Plot 10 (SPSS, USA); así mismo, se efectuó el análisis de varianza y prueba de comparación mediante la LSD de Fisher a un $5 \%$ de nivel de significancia mediante el programa Statgraphics centurión (c) Plus versión XV.II. La bondad de ajuste del modelo se evaluó con los parámetros $R^{2}$ (coeficiente de determinación), \% E (error porcentual promedio) ecuación 7 y RMSE (raíz cuadrada del error medio cuadrático) ecuación 8.

$$
\begin{aligned}
& \% \mathrm{E}=\frac{\sum_{\mathrm{i}=1}^{\mathrm{n}} \frac{\mid \text { Ccal-Cexp|n }}{\operatorname{Cexp} \mathrm{n}}}{\mathrm{n}} \cdot 100 \\
& \mathrm{RMSE}=\left[\frac{\sum_{\mathrm{i}=1}^{\mathrm{n}(\text { Ccal-Cexp })^{2}}}{\mathrm{n}}\right]^{0.5}
\end{aligned}
$$

Donde: Ccal: valor calculado; Cexp: valor experimental; $n$ : número de determinaciones. Según Brousse et al., (2012) un buen ajuste es indicado por valores pequeños de RMSE, $R^{2}>0.85$ y E $\%<10 \%$.

\section{RESULTADOS Y DISCUSION}

Para mayor claridad, los resultados son presentados y discutidos en cinco subsecciones: cinéticas de pérdida de agua, estudio de la pérdida de agua como modelo difusivo, energías de activación y modelación matemáticas de las curvas de pérdida de agua

\section{Cinéticas de pérdida de agua}

Según Bravo (2008) tanto en el proceso de fritura al vacío como fritura convencional, las curvas de pérdida de agua son semejantes a las curvas del clásico perfil de secado. En las figura 1 se muestra el comportamiento cinético de la pérdida de agua en las semillas de arveja (Pisum sativum L.) de la variedad Obonuco Andina durante la fritura; demostrando contenidos iniciales de humedad del $68 \%$ y reducciones significativas al final de los procesos (240 segundos) bajo las diferentes condiciones de estudio (presiones y temperaturas) en los siguientes porcentajes: T1a (81.13 \pm 6.56$)$, T1b (91.58 \pm 8.76$), \mathrm{T} 1 \mathrm{c}(95.36 \pm 7.45), \mathrm{T} 2 \mathrm{~b}$ (88.89 \pm 9.56), T2b (92.56 \pm 5.89), T2c (93.33 \pm 11.21$)$, T3a (92.20 \pm 9.89$),$ T3b (96.19 \pm 5.78$),$ T3c (97.37 \pm $6.87)$.

Los resultados permiten afirmar que a mayor temperatura mayor es la pérdida de humedad; Debnath et al., (2003) reportan conclusión similar. No obstante, a mayor vacío mayor fue la pérdida de agua, debido a que la reducción de presión, ocasiona disminución en los puntos de ebullición; a mayor vacío estos puntos se alcanzan con mayor facilidad (Shyu et al., 2005) en consecuencia, el agua albergada en el producto empezará a vaporizarse más rápidamente (Bravo, 2008).

\section{Estudio de la pérdida de agua como modelo difusivo.}

En la tabla 1 se indican los valores de difusividad (Deff) para cada presión y temperatura, mostrando la dependencia de la fritura con los dos factores. Los valores encontrados de difusividad efectiva, se encuentran dentro del intervalo general de $10^{-8}$ y $10^{-11}$ para deshidratación de alimentos (Sacilik y Elicin, 2006). Al comparar los tratamientos, se evidenciaron valores de difusividad mayores a menores presiones y temperaturas más elevadas. Bravo (2008) afirma que cuanto mayor es el grado de vacío y mayor la temperatura, la velocidad de evaporación de agua aumenta aumentando la velocidad de secado y disminuyendo el tiempo de fritura. En el estudio de Troncoso y Pedreschi (2009) los resultados indicaron que durante la fritura al vacío de rodajas de papa pre-tratada, la temperatura más alta de fritura mostró los 
menores tiempos de fritura, afirmado que los tratamientos a vacío fueron menores comparados con los de presión atmosférica. Así mismo, la humedad del producto fue significativamente afectada durante los primeros $120 \mathrm{~s}$ de fritura a vacío a $130^{\circ} \mathrm{C}$, similar a los resultados obtenidos en esta investigación cuando se redujo la presión hasta $9 \mathrm{kPa}$ el efecto se observó en los primeros $150 \mathrm{~s}$.
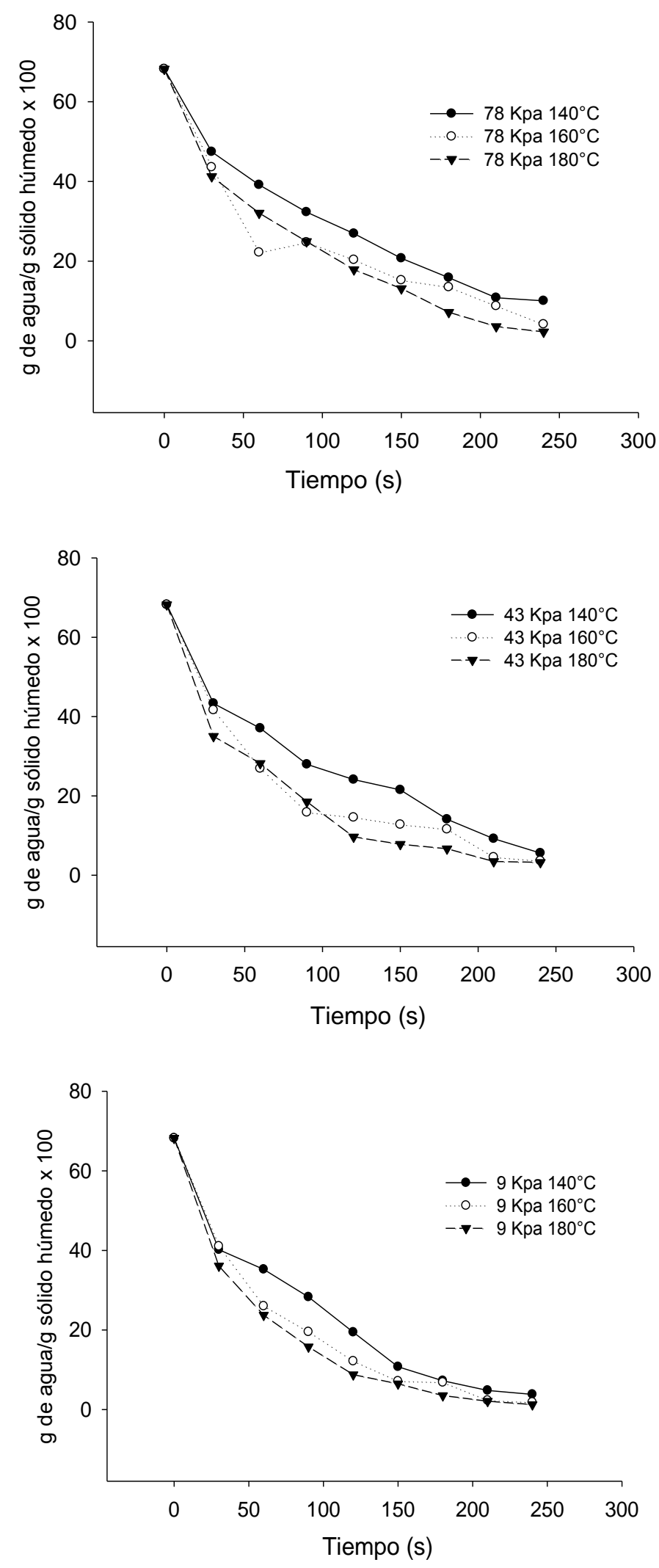

Fig. 1. Cinéticas de pérdida de humedad a diferentes condiciones de proceso. 
Según el análisis de varianza (ANOVA) existe diferencias estadísticamente significativas entre los valores de difusividad en las tres temperaturas en estudio $(p$-valor $<0.05)$ sin tener en cuenta la presión. Sin embargo, al comparar las presiones con cada una de las temperaturas en estudio, se determinó equivalencias entre algunos tratamientos. Un tratamiento a $78 \mathrm{kPa}$ con $160^{\circ} \mathrm{C}$ es similar a uno de $43 \mathrm{kPa}$ con $140^{\circ} \mathrm{C}$ (tabla 1$)$.

Tabla 1. Parámetros difusiónales de agua, en proceso de fritura de arveja. *Valores promedio $(n=5) \pm$ desviación estándar; *Letras no comunes implican diferencias entre promedios, según prueba de LSD de Fisher aun $95 \%$ de confianza.

\begin{tabular}{|c|c|c|c|c|}
\hline Presión (kPa) & Temperatura $\left({ }^{\circ} \mathrm{C}\right)$ & ${ }^{*} D_{\text {eff }} \times 10^{-8}\left(\mathrm{~m}^{2} \mathrm{~s}^{-1}\right)$ & ${ }^{*} D o \times 10^{-6}\left(\mathrm{~m}^{2} \mathrm{~s}^{-1}\right)$ & $R^{2}$ \\
\hline \multirow{3}{*}{78} & 140 & $1.62 \pm 0.12 \mathrm{a}$ & \multirow{3}{*}{$4.08 \pm 0.32$} & \multirow{3}{*}{0.97} \\
\hline & 160 & $2.23 \pm 0.16 b c$ & & \\
\hline & 180 & $2.61 \pm 0.23 \mathrm{de}$ & & \\
\hline \multirow{3}{*}{43} & 140 & $2.01 \pm 0.24 b$ & \multirow{3}{*}{$0.925 \pm 0.13$} & \multirow{3}{*}{0.99} \\
\hline & 160 & $2.59 \pm 0.27 \mathrm{~cd}$ & & \\
\hline & 180 & $2.86 \pm 0.16$ ef & & \\
\hline \multirow{3}{*}{9} & 140 & $2.42 \pm 0.18 \mathrm{~cd}$ & \multirow{3}{*}{$1.28 \pm 0.11$} & \multirow{3}{*}{0.98} \\
\hline & 160 & $3.02 \pm 0.22 f$ & & \\
\hline & 180 & $3.44 \pm 0.31 \mathrm{~g}$ & & \\
\hline
\end{tabular}

\section{Energías de activación}

La energía de activación se determinó a partir de la pendiente de la figura 2 (grafico de Arrhenius) los valores obtenidos fueron: $18.67 \pm 0.93 \mathrm{~kJ} / \mathrm{mol}, 13.82 \pm 0.87 \mathrm{~kJ} / \mathrm{mol}$ y $13.75 \pm 1.06 \mathrm{~kJ} / \mathrm{mol}$, para $78 \mathrm{kPa}, 43$ $\mathrm{kPa}$ y $9 \mathrm{kPa}$, respectivamente. Según Melquíades et al., (2009) estos valores, termodinámicamente representan la energía que necesitan las moléculas de agua para su migración o movimiento dentro del producto.

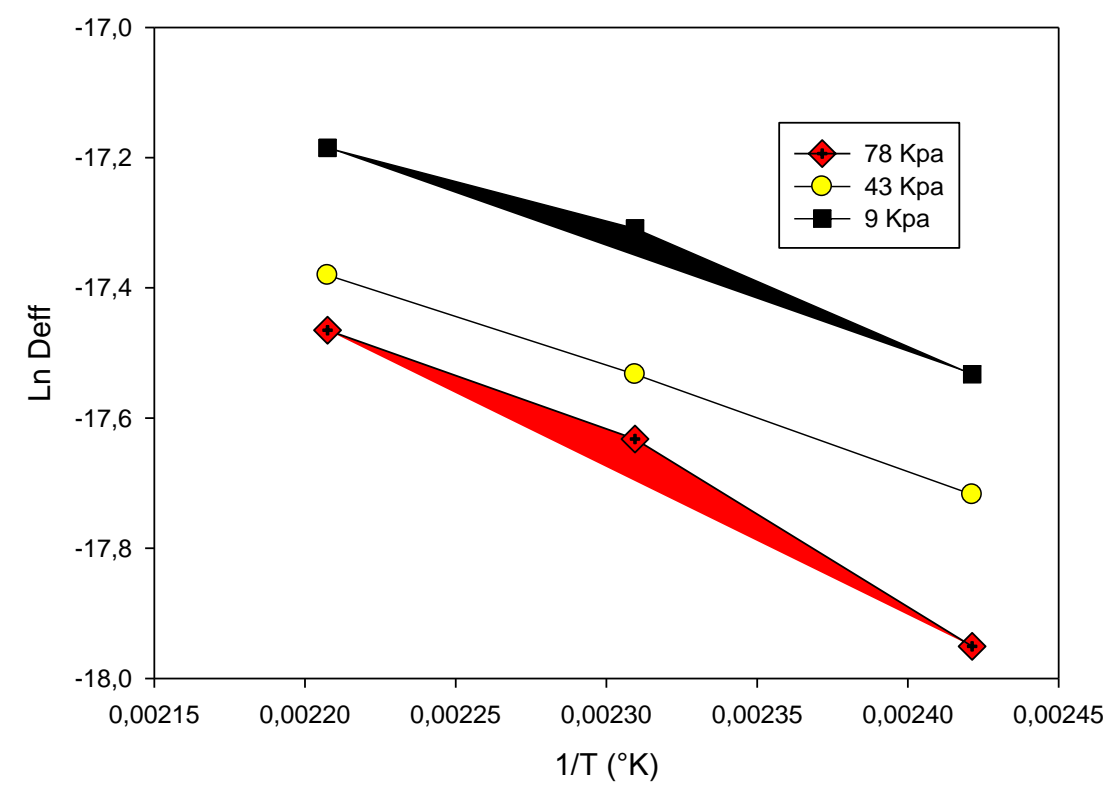

Fig. 2. Gráfico de Arrhenius: $78 \mathrm{kPa}\left(\mathrm{y}=-2245.9 \mathrm{x}-12.484 \mathrm{R}^{2}=0.97\right), 43 \mathrm{kPa}(\mathrm{y}=-1663.4 \mathrm{x}-$ 13.678 $\left.\mathrm{R}^{2}=0.95\right), 9 \mathrm{kPa}\left(\mathrm{y}=-1654.2 \mathrm{x}-13.52 \mathrm{R}^{2}=0.98\right)$.

La energía de activación de este fenómeno proviene inicialmente de la energía térmica aportada por el fluido (aceite) observando que a mayor temperatura, se requiere de menor Ea para iniciar el proceso; así mismo, se puedo observar que a medida que se hace vacío al sistema, se requiere de menor Ea para dar inicio al mismo proceso, con lo cual se concluye que a mayor vacío y mayor temperatura la Ea es menor para comenzar la evaporación del agua interna del producto (Bravo, 2008). No obstante, al comparar los valores de energía de activación se encontró diferencias estadísticamente significativas ( $p$-Valor $<0.05)$ entre el tratamiento a $78 \mathrm{kPa}$ frente a los de 43 y $9 \mathrm{kPa}$, contrario a lo reportado por Bravo (2008) quien menciona que la energía de enlace del agua con la matriz vegetal, así como el calor latente de vaporización varían poco con la presión, explicando el no haber encontrado diferencias significativas en su estudio. Sin 
embargo, una posible explicación del fenómeno observado seria la diferencia de los materiales vegetales empleados en dichas investigaciones. En el estudio de Bravo (2008) se empleó rodajas de Manzana, donde el frente de exposición (mesocarpio o pulpa) al medio de fritura está totalmente dispuesto para el proceso de deshidratación. Mientras que en esta investigación la leguminosa objeto de estudio presenta una cutícula (testa) que recubre los cotiledones ocasionando un efecto barrera al proceso de deshidratación.

De otra parte, es importante señalar que los productos alimenticios y en este caso la arveja es susceptible a las altas temperaturas por largos tiempos de proceso muchos, por ello es necesario estudiar algunos parámetros de calidad asociados a estos fenómenos de transferencia de masa y calor como son: calidad sensorial, color, textura, entre otros.

\section{Modelación matemáticas de las curvas de pérdida de agua}

El ajuste de los datos experimentales mostró que el modelo Page (tablas 2) describe mejor la pérdida de agua en las semillas de arveja de la variedad Obonuco Andina, como lo muestran los valores de $R^{2}$, RMSE y E \%; no obstante, los modelos de Newton y Henderson-Pabis (tabla 3 y 4) son buenas opciones para describir la pérdida de agua durante la operación de fritura. Garg et al., (2014), Da Rocha et al., (2012) y Martínez et al., (2010) reportaron datos similares afirmando que el modelo Page proporciona los mejores resultados de ajuste en sus estudios.

Tabla 2. Parámetros cinéticos y bondad de ajuste del modelo de Page. En la tabla, *Valores promedio $(n=5) \pm$ desviación estándar. *Letras no comunes implican diferencias entre según prueba de LSD de Fisher aun 95\% de confianza.

\begin{tabular}{|c|c|c|c|c|c|c|}
\hline \multirow{2}{*}{ Presión (kPa) } & \multirow{2}{*}{ Temperatura $\left({ }^{\circ} \mathrm{C}\right)$} & ${ }^{*} k_{1} \times 10^{-2}\left(\mathrm{~s}^{-1}\right)$ & \multirow{2}{*}{${ }^{*} n$} & \multicolumn{3}{|c|}{ Bondad de ajuste } \\
\cline { 3 - 7 } & & & & \multicolumn{2}{|c|}{$\mathrm{RSME}$} & $\mathrm{E} \%$ \\
\hline \multirow{3}{*}{78} & 140 & $1.71 \pm 0.17 \mathrm{a}$ & $0.85 \pm 0.08$ & 0.99 & 0.05 & 1.46 \\
\cline { 2 - 7 } & 160 & $1.92 \pm 0.42 \mathrm{ab}$ & $0.69 \pm 0.07$ & 0.97 & 0.42 & 12.53 \\
\cline { 2 - 7 } & 180 & $2.14 \pm 0.19 \mathrm{bc}$ & $0.88 \pm 0.08$ & 0.98 & 0.28 & 8.03 \\
\hline \multirow{3}{*}{43} & 140 & $2.49 \pm 0.23 \mathrm{c}$ & $0.79 \pm 0.08$ & 0.98 & 0.33 & 9.40 \\
\cline { 2 - 7 } & 160 & $4.20 \pm 0.24 \mathrm{e}$ & $0.78 \pm 0.04$ & 0.99 & 0.27 & 7.54 \\
\hline \multirow{3}{*}{9} & 180 & $1.92 \pm 0.21 \mathrm{ab}$ & $0.89 \pm 0.10$ & 0.98 & 0.36 & 9.96 \\
\cline { 2 - 7 } & 140 & $2.28 \pm 0.17 \mathrm{bc}$ & $0.90 \pm 0.08$ & 0.99 & 0.25 & 6.91 \\
\cline { 2 - 7 } & 160 & $3.34 \pm 0.22 \mathrm{~d}$ & $0.85 \pm 0.06$ & 0.99 & 0.21 & 6.39 \\
\hline
\end{tabular}

Tabla 3. Parámetros cinéticos y bondad de ajuste del modelo de Newton. *Valores promedio $(n=5) \pm$ desviación estándar. *Letras no comunes implican diferencias entre promedios, según prueba de LSD de Fisher aun 95\% de confianza.

\begin{tabular}{|c|c|c|c|c|c|}
\hline \multirow{2}{*}{ Presión (kPa) } & \multirow{2}{*}{ Temperatura $\left({ }^{\circ} \mathrm{C}\right)$} & \multirow{2}{*}{${ }^{*} k_{2} \times 10^{-2}\left(\mathrm{~s}^{-1}\right)$} & \multirow{2}{*}{$R^{2}$} & \multicolumn{2}{|c|}{ Bondad de ajuste } \\
\hline & & & & RSME & $E \%$ \\
\hline \multirow{3}{*}{78} & 140 & $0.82 \pm 0.10 a$ & 0.98 & 0.28 & 8.03 \\
\hline & 160 & $1.06 \pm 0.12 a b c$ & 0.92 & 0.53 & 15.00 \\
\hline & 180 & $1.31 \pm 0.13 \mathrm{cde}$ & 0.96 & 0.48 & 13.60 \\
\hline \multirow{3}{*}{43} & 140 & $0.94 \pm 0.15 a b$ & 0.96 & 0.44 & 12.48 \\
\hline & 160 & $1.22 \pm 0.13$ cde & 0.94 & 0.51 & 14.48 \\
\hline & 180 & $1.38 \pm 0.14 \mathrm{de}$ & 0.96 & 0.39 & 10.90 \\
\hline \multirow{3}{*}{9} & 140 & $1.21 \pm 0.17 \mathrm{bcd}$ & 0.98 & 0.40 & 10.43 \\
\hline & 160 & $1.49 \pm 0.23$ ef & 0.98 & 0.23 & 6.59 \\
\hline & 180 & $1.66 \pm 0.21 \mathrm{f}$ & 0.99 & 0.17 & 4.91 \\
\hline
\end{tabular}

Los valores de $\mathrm{R}^{2}$ superiores a 0.95 para los valores de las constantes cinéticas de velocidad de pérdida de agua $\mathrm{k}_{1}$, $\mathrm{k}_{2}$ y $\mathrm{k}_{3}$ demuestran dependencia con la temperatura (Vega, Tello y Lemus, 2007). Al comparar las constantes entre si se encontraron diferencias estadísticamente significativas ( $p$-Valor=0.0001). Para el caso del parámetro k1 del modelo de Page, éste aumenta su valor al aumentar la temperatura del aceite. Martínez et al., (2010) presenta datos similares donde no se evidencia correlación de las K conforme aumentó la temperatura utilizando el modelo de Page. Azzouz et al., (2002) mencionan que este parámetro 
está en función de la temperatura del medio calefactor y el contenido de humedad inicial. No obstante los valores de $\mathrm{K}_{1}$ no muestran correlación de aumento conforme disminuyó la presión. En la figura 1 se observa que bajo condiciones de presión de $9 \mathrm{kPa}$, la humedad en equilibrio se logra a menor tiempo debido a la disminución en los puntos de ebullición, esto ocasiona que el grafico cinético comience a perder pendiente para tornarse constante, explicando el fenómeno observado en la tabla 2. Sin embargo, bajo los modelos de Newton y Henderson - Pabis se nota aumento de los valores de $\mathrm{k}_{2}$ y $\mathrm{K}_{3}$ conforme se incrementó la temperatura y disminuyó la presión.

Tabla 4. Parámetros cinéticos y bondad de ajuste del modelo de Henderson - Pabis. *Valores promedio $(n=5) \pm$ desviación estándar. *Letras no comunes implican diferencias entre promedios, según prueba de LSD de Fisher aun $95 \%$ de confianza.

\begin{tabular}{|c|c|c|c|c|c|c|}
\hline \multirow{2}{*}{$\begin{array}{c}\text { Presión } \\
(\mathrm{kPa})\end{array}$} & Temperatura $\left({ }^{\circ} \mathrm{C}\right)$ & \multirow{2}{*}{${ }^{*} \mathrm{k}_{3} \times 10^{-2}\left(\mathrm{~s}^{-1}\right)$} & \multirow{2}{*}{${ }^{*} \mathrm{a}$} & \multicolumn{3}{|c|}{ Bondad de ajuste } \\
\cline { 3 - 7 } & & & & \multicolumn{2}{|c|}{$R S M E$} & \multicolumn{2}{c|}{$R^{2}$} \\
\hline \multirow{3}{*}{78} & 140 & $0.80 \pm 0.11 \mathrm{a}$ & $0.96 \pm 0.09$ & 0.99 & 0.23 & 6.57 \\
\cline { 2 - 7 } & 160 & $0.90 \pm 0.10 \mathrm{ab}$ & $0.87 \pm 0.08$ & 0.95 & 0.39 & 11.93 \\
\cline { 2 - 7 } & 180 & $1.30 \pm 0.19 \mathrm{def}$ & $1.11 \pm 0.08$ & 0.97 & 0.37 & 11.36 \\
\hline \multirow{3}{*}{43} & 140 & $0.90 \pm 0.12 \mathrm{ab}$ & $0.99 \pm 0.09$ & 0.97 & 0.42 & 11.93 \\
\cline { 2 - 7 } & 160 & $1.10 \pm 0.09 \mathrm{bc}$ & $0.86 \pm 0.04$ & 0.95 & 0.34 & 9.64 \\
\hline \multirow{3}{*}{9} & 180 & $1.40 \pm 0.11 \mathrm{cde}$ & $0.84 \pm 0.11$ & 0.98 & 0.25 & 7.13 \\
\cline { 2 - 7 } & 140 & $1.20 \pm 0.16 \mathrm{~cd}$ & $1.03 \pm 0.10$ & 0.98 & 0.30 & 8.18 \\
\cline { 2 - 7 } & 160 & $1.50 \pm 0.17 \mathrm{ef}$ & $1.01 \pm 0.10$ & 0.98 & 0.24 & 6.77 \\
\hline
\end{tabular}

Respecto del parámetro $n$ del modelo de Page y el parámetro a del modelo de Henderson-Pabis (tabla 3 y 4), no presentaron dependencia con la temperatura, considerándose como parámetros constantes. Algunos autores citados por Vega, Tello y Lemus (2007) proponen que valores del parámetro $n$ de Page dependen del tipo de producto que se va a deshidratar y no de la temperatura de secado.

\section{CONCLUSIONES}

Los modelos empíricos utilizados (Newton, Henderson-Pabis y Page) mostraron una buena calidad de ajuste sobre las curvas experimentales, validando su utilización. No obstante, el modelo de Page describe mejor el fenómeno observado.

La presión y la temperatura mostraron tener influencia estadísticamente significativa sobre las constantes cinéticas de pérdida de agua en las semillas de arveja (Pisum sativum L.), permitiendo calcular valores de energía de activación 18.67, 13.82 y 13.75 kJ/mol, para 78, 43 y 9 kPa respectivamente.

Los valores encontrados de difusividad efectiva, se encuentran dentro del intervalo general de $10^{-8}$ y $10^{-11}$ para deshidratación de alimentos.

\section{AGRADECIMIENTOS}

Al Sistema General de Regalías por el aporte de los recursos en el proyecto BPIN 2012000100188 con el cual se realizaron parte de los estudios. A las universidades de San Buenaventura de Cali y a la Universidad de Caldas, a los docentes y directivas involucradas en este proceso de investigación. A la Vicerrectoría de Investigaciones de la Universidad de Nariño por el apoyo económico en la financiación dell Banco proyectos docentes de Investigación Científica, Desarrollo Tecnológico, Innovación y Creación Artística, 2014.

\section{REFERENCIAS}

Alvis, A. y C. Vélez, C, Modelado del proceso de fritura del Ñame (Dioscorea alata) mediante mediciones reológicas usando la metodología de superficie de respuesta, doi: 10.1612/inf.tecnol.3958ait.07, Inf. Tecnol. 19 (5), 11-18 (2008)

Alvis, A., H. Villada, y D. Villada, Efecto de la temperatura y tiempo de fritura sobre las características sensoriales del Ñame (Dioscorea alata), doi:10.1612/inf.tecnol.3958bit.07, Inf. Tecnol. 19 (5), 19-26 (2008)

Amarakoon, D., D. Thavarajah, K. McPhee, y P. Thavarajah. Iron-, zinc-, and magnesium-rich field peas (Pisum sativum L.) with naturally low phytic acid: A potential food-based solution to global micronutrient malnutrition, doi:10.1016/j.jfca.2012.05.007, J. Food. Compos. Anal, 27, 8-13 (2012) 
Azzouz, S., A. Guizani, W. Jomaa, y A. Belghith, Moisture diffusivity and drying kinetic equation of convective drying of grapes, doi: 10.1016/S0260-8774(02)00109-7, J. Food. Eng. 55 (4), 323-330 (2002)

Banga, J., E. Balsa-Canto, C. Moles, y A. Alonso, Improving food processing using modern optimization methods, doi: 10.1016/S0924-2244(03)00048-7, Trends. Food. Sci. Tech. 14 (4), 131-144 (2003)

Bernal De, R. I., Análisis de alimentos. 3ª edición, 58-60. Editora Guadalupe Ltda, Bogotá, Colombia (1998)

Bravo, J, Contribución al estudio de la fritura al vacío: Deshidratación de rojadas de manzana, Tesis de Doctorado en Tecnología de Alimentos, Universidad Politécnica de Valencia, España (2008)

Brousse, M. M., A. B. Nieto, A. R. Linares, y M. L. Vergara. Cinética de adsorción de agua en purés deshidratados de mandioca (Manihot esculenta Crantz), http://www.rvcta.org; ISSN: 2218-4384, Rev. Venez. Cienc. y Tecnol. Aliment.3 (1), 80-96 (2012)

Costa, R., y F. Oliveira, Modelling the kinetics of water loss during potato frying with a compartmental dynamic model, doi: 0.1016/S0260-8774(99)00095-3, J. Food. Eng.. 41, 177- 85 (1999).

Da Rocha, R., E. Melo, J. Corbín, P. Berbert, S. Donzeles, J. Tabar, Cinética del secado de tomillo, doi: 10.1590/S1415-43662012000600013, R. Bras. Eng. Agríc., 16(6), 675-683 (2012)

Da Silva, P., y G. Moreira, Vacuum frying of high-quality fruit and vegetable-based snacks, doi: 10.1016/j.Iwt.2008.01.016, LWT-Food. Sci. Technol., 41, 1758 - 1767 (2008)

Debnath, S., K. Bhat, y N. Rastogi, Effect of pre-drying on kinetics of moisture loss and oil uptake during deep fat frying of chickpea flour-based snack food, LWT - Food. Scie. Technol., 36, 91-98 (2003)

Dobarganes, C., G. Márquez-Ruiz, y J. Velasco, Interactions between fat and food during deep-frying, doi: 10.1002/1438-9312(200009)102:8/9<521::AID-EJLT521>3.0.CO;2-A, Eur. J. Lipid. Sci. Tech., 102, 521-528 (2000)

Espinosa, L.E., M. Pérez, M. T. Martínez, R. Castro, y G. Barrios, Efecto de empaques y temperaturas en el almacenamiento de chile manzano (Capsicum pubescens Ruíz y Pavón), http://www.scielo.org.mx/scielo.php?script=sci_arttext\&pid=S1027-152X2010000200007, ISSN 20074034, Rev. Chapingo. Ser. Hort., 16(2), 115-121 (2010)

Fenalce, El cultivo de la arveja, historia y su importancia (2010), http://www.fenalce.org/arch_public/arveja93.pdf. Acceso: 10 de junio (2015)

Garg, M., S. Sharma, S. Varmani, y S. Sadhu, Drying kinetics of thin layer pea pods using tray drying, http://www.ijfans.com/volume\%203\%20issue\%203/12.pdf, ISSN: 2320-7876, IJFNS, 3(3), 61-66 (2014)

Gómez, S, Efecto de las condiciones de fritura a vacío en el procesado de chips de kiwi. Tesis de Master en Ingeniería de Alimentos, Universidad Politécnica de Valencia, España (2012)

Kochhar, S. P., y G. Gertz, New theoretical and practical aspects of the frying process, doi: 10.1002/ejlt.200400996, Eur. J. Lipid. Sci. Tech., 106 (11), 722-727 (2004)

Martínez, G., A. Flores, J. Mercado, M. López, Características de secado de nopal (Opuntia ficus-indica) por lecho fluidizado, http://www.actauniversitaria.ugto.mx/index.php/acta/article/viewFile/61/48, ISSN: 0188 6266, Acta. Univ., 20 (Número Especial 3), 70-76 (2010)

Melquíades, Y. I., C. López, M. E. Rosas. Estudio de la cinética de rehidratación de zanahoria (Daucus Carota) deshidratadas, doi: 10.1612/inf.tecnol.4037it.08, Inf. Tecnol., 20(3), 65-72 (2009)

NTC 3272: Norma Técnica Colombiana sobre grasas y aceites comestibles para frito industrial, 6, Bogotá, Colombia (1997)

NTC 1250: Norma Técnica Colombiana sobre alverja verde, 1-4, Bogotá, Colombia (1979)

Osorio, L., y M. Castaño, Caracterización del agente causante de la pudrición de raíces de la arveja (Pisum sativum linneo), enfermedad endémica en el municipio de Manizales Caldas (Colombia), http://200.21.104.25/agronomia/downloads/Agronomia19(2)_4.pdf, ISSN: 0568-3076, Agron, 19(2), 33-43 (2011) 
Osorio, O., Plan estratégico departamental en CT+l: Capítulo 5.5.3.5 demanda de ciencia y tecnología en la cadena hortofrutícola (2012), http://www.ccpasto.org.co/index.php/planes-estrategicos. Acceso: 4 de junio (2015)

Pabón, J., y J. Castaño, Manejo De La Pudrición Radical De La Arveja (Pisum Sativum Linneo) Causada Por Fusarium oxysporu, http://200.21.104.25/agronomia/downloads/Agronomia20(2)_5.pdf, ISSN: 05683076, Agron, 20(2), 37-44 (2012)

Sacilik, K., y A. Elicin, The thin layer drying characteristics of organic apple slices, doi: 10.1016/j.jfoodeng.2005.03.024, J. Food. Eng., 73(3), 281-289 (2006)

Shyu, S. L., B. L Hau, y L. S. Hwang, Effects of processing conditions on the quality of vacuum-fried carrot chips, doi: 10.1002/jsfa.2195, J. Sci. Food. Agr., 85 (11), 1903-1908 (2005)

Téllez-Pérez, C., M. M. Sabah, J. G. Montejano-Gaitán, V. Sobolik, C. A. Martínez, y K. Allaf, Impact of instant controlled pressure drop treatment on dehydration and rehydration kinetics of green Moroccan pepper (Capsicum annuum), doi: 10.1016/j.proeng.2012.07.491, Procedia. Eng., 42, 978-1003 (2012)

Troncoso, E., y F. Predreschi, Modeling water loss and oil uptake during vacuum frying of pre-treated potato slices, doi:10.1016/j.Iwt.2009.01.008, LWT - Food. Scie. Technol., 42, 1164-1173 (2009)

Vega, A., C.Tello, y R. Lemus, Continuous measurement of convective heat flux during deep-frying: validation and application to inverse modeling. doi: 10.4067/S0718-33052007000100008, Ingenieare. Rev. Chil. Ing., 15 (1), 55-64 (2007)

Vitrac, 0., G. Trystram, y A. Raoult-Wack, Continuous measurement of convective heat flux during deepfrying: validation and application to inverse modeling, doi: 10.1016/S0260-8774(03)00024-4, J. Food. Eng., $60(2), 111-124(2003)$ 